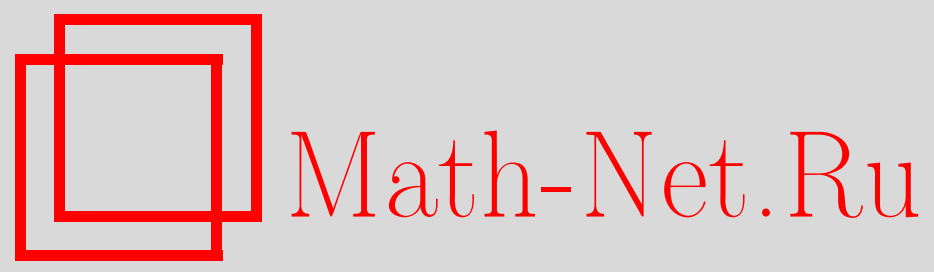

О. В. Черемисин, Об активности схем из клеточных элементов, реализующих систему всех конъюнкций, Дискрет. матем., 2003, том 15, выпуск 2, 113-122

DOI: https://doi.org/10.4213/dm198

Использование Общероссийского математического портала Math-Net.Ru подразумевает, что вы прочитали и согласны с пользовательским соглашением http://www. mathnet.ru/rus/agreement

Параметры загрузки:

IP : 54.224 .60 .19

26 апреля 2023 г., 05:44:46

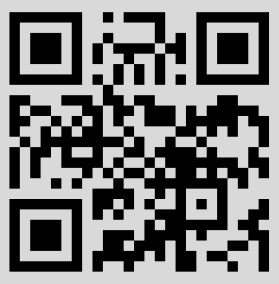




\title{
Об активности схем из клеточных элементов, реализующих систему всех конъюнкций
}

\author{
( 2003 г. О. В. Черемисин
}

\begin{abstract}
Изучается активность схем из клеточных элементов, мера сложности, характеризующая функционирование схем с энергетической точки зрения. Для системы $K_{n}$ всех элементарных конъюнкций $n$ переменных при $n \rightarrow \infty$ найден порядок роста минимальной активности схем из клеточных элементов, реализующих $K_{n}$. Доказана невозможность одновременного достижения минимального по порядку роста активности и сложности реализации системы $K_{n}$ в указанном классе схем.
\end{abstract}

В работе изучается активность схем из клеточных элементов, реализующих систему $K_{n}$ всех элементарных конъюнкций от $n$ переменных, то есть систему всех булевых функций вида $x_{1}^{\sigma_{1}} \ldots x_{n}^{\sigma_{n}}$, где $\sigma_{1}, \ldots, \sigma_{n} \in\{0,1\}$.

Понятие схемы из клеточных элементов введено в [1]. В этой работе найден порядок роста функции Шеннона $L(n)$ сложности реализации булевых функций схемами из клеточных элементов, показано, что

$$
\frac{1}{4} 2^{n} \lesssim L(n) \lesssim \frac{9}{2} 2^{n}
$$

при $n \rightarrow \infty$. Позднее, в [2] было доказано существование асимптотики для $L(n)$. Точные по порядку оценки сложности реализации некоторых конкретных систем функций схемами из клеточных элементов были получены в $[3,4]$.

Рассматриваемое в данной работе понятие активности схем из клеточных элементов аналогично понятию мощности схем из функциональных элементов, введенному в [5]. Дальнейшее исследование мощности схем из функциональных элементов было проведено О. М. Касим-Заде [6]. В [7] О. М. Касим-Заде доказана возможность одновременного достижения порядка роста функции Шеннона для мощности и асимптотики функции Шеннона для сложности при реализации булевых функций схемами из функциональных элементов в базисе $\{\&, \vee,-$

Все необходимые определения, касающиеся схем из клеточных элементов, можно найти в $[1,4]$. Кратко повторим основные из них.

Рассмотрим шесть базисных элементов: три функциональных (рис. 1) и три коммутационных (рис. 2). Каждый из этих элементов имеет форму единичного квадрата и может быть повернут на угол $k \pi / 2, k=0,1,2,3$.

Функциональным элементам ставятся в соответствие обычные функциональные элементы (рис. 3). Первые два коммутационных элемента служат для соединения функциональных -элементов (они являются аналогами физических проводников электрического 


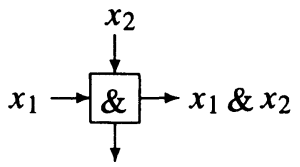

$x_{1} \& x_{2}$

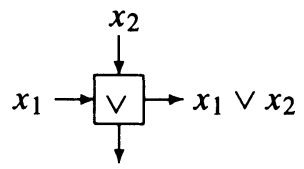

$x_{1} \vee x_{2}$<smiles>[Y4][Y]1=C([Y])C([Z1])C1[3H]</smiles>

$\bar{x}_{2}$

Рис. 1.

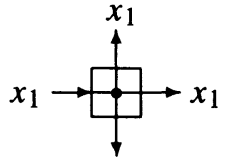

$x_{1}$

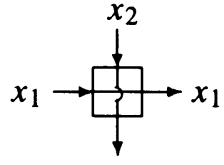

$x_{2}$

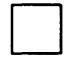

Рис. 2.

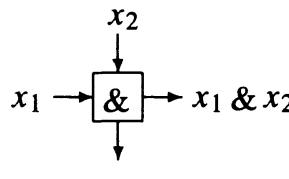

$x_{1} \& x_{2}$

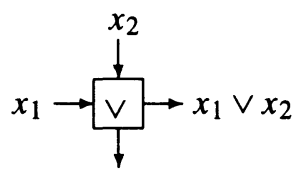

$x_{1} \vee x_{2}$<smiles>[Y2][Y]1=C([Al])C([Z1])C1[Y2]</smiles>

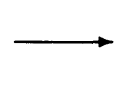

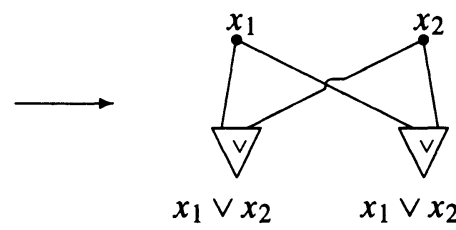
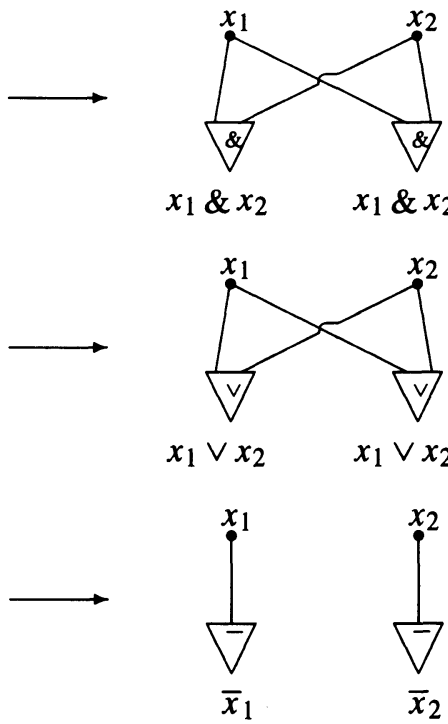

$x_{1} \& x_{2} \quad x_{1} \& x_{2}$

Рис. 3. 


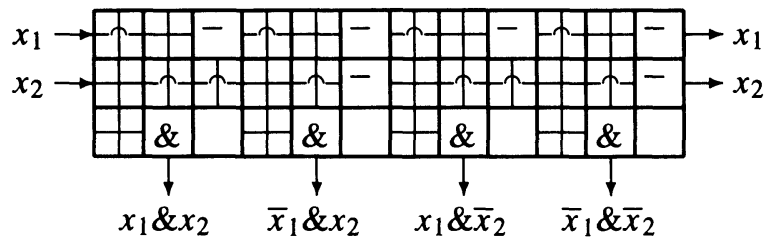

Рис. 4.
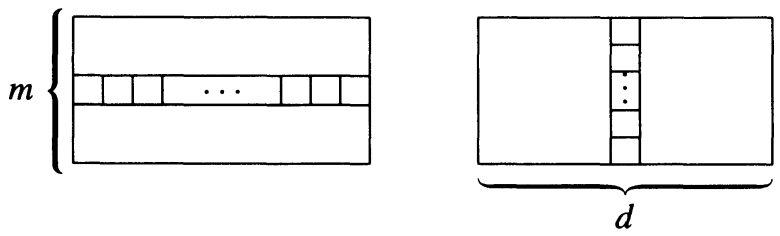

Рис. 5.

тока). На третий элемент могут подаваться как входы, так и выходы других элементов (он является аналогом изолятора электричества).

Схемой из клеточных элементов называется прямоугольная система клеточных элементов такая, что при замене клеточных функциональных элементов (рис. 1) на обычные функциональные элементы в соответствии с рис. 3 и соединении последних в соответствии с тем, что предписывают клеточные коммутационные элементы, получается обычная схема из функциональных элементов, удовлетворяющая известному определению, данному в [8].

Для наглядности на рис. 4 приведен пример схемы из клеточных элементов, реализующей систему всех конъюнкций двух переменных.

В дальнейшем будем также называть клеточные элементы ячейками схемы. Горизонтальной (вертикальной) цепочкой ячеек в схеме из клеточных элементов будем называть всякую последовательность элементов, расположенных вдоль некоторой горизонтальной (вертикальной) прямой так, что любые два соседних элемента этой последовательности имеют общую сторону, а крайние элементы этой последовательности примыкают к сторонам схемы (рис. 5).

Число горизонтальных (вертикальных) цепочек ячеек в схеме называется шириной (длиной) этой схемы. Ширина (длина) схемы $S$ обозначается через $m(S)$ (соответственно, $d(S))$.

Под сложностью схемы $S$ понимается ее площадь (число элементов). Эта величина обозначается через $L(S)$. Очевидно, что $L(S)=m(S) d(S)$.

Сложностью системы $K_{n}$ называется величина

$$
L\left(K_{n}\right)=\min _{S} L(S),
$$

где минимум берется по всевозможным схемам $S$, реализующим систему $K_{n}$.

Теперь дадим определение активности схем из клеточных элементов. Пусть $S$ - произвольная схема. Для произвольного набора $\tilde{\alpha}=\left(\alpha_{1}, \ldots, \alpha_{n}\right)$ значений переменных, при- 
писанных входам схемы $S$, обозначим через $E(S, \tilde{\alpha})$ число элементов схемы, как функциональных, так и коммутационных, у которых хотя бы один выход находится в состоянии 1 при поступлении на входы схемы набора $\tilde{\alpha}$. Величину $E(S, \tilde{\alpha})$ назовем акгивностью схемы $S$ на наборе $\tilde{\alpha}$.

Максимальной активностью схемы $S$ назовем величину

$$
E_{\max }(S)=\max _{\tilde{\alpha}} E(S, \tilde{\alpha}) .
$$

Средней активностью схемы $S$ назовем величину

$$
E_{\mathrm{m}}(S)=\frac{1}{2^{n}} \sum_{\tilde{\alpha}} E(S, \tilde{\alpha}) .
$$

Максимальной (средней) активностью системы всех конъюнкций $K_{n}$ назовем величину

$$
E_{\max }\left(K_{n}\right)=\min _{S} E_{\max }(S)
$$

(соответственно,

$$
\left.E_{\mathrm{m}}\left(K_{n}\right)=\min _{S} E_{\mathrm{m}}(S)\right)
$$

(в обоих случаях минимум берется по всевозможным схемам $S$, реализующим систему $\left.K_{n}\right)$.

Для введенного описанным образом понятия активности схем из клеточных элементов, как и для аналогичного понятия мощности схем из функщиональных элементов, возможны несколько содержательных интерпретаций.

Наиболее известная из них - тепловая интерпретация. При этой интерпретации считается, что элементы схемы способны выделять тепло, причем элемент (функщиональный или коммутационный) выделяет тепло в том и только том случае, когда хотя бы один из его выходов находится в состоянии 1 , и всякий элемент, выделяющий тепло, выделяет единицу тепла за единицу времени. При этом величины $E_{\max }(S)$ и $E_{\mathrm{m}}(S)$ интерпретируются соответственно как максимальная и средняя тепловые мощности схемы.

С. С. Кравцовым [1] для всякого $n, n \geqslant 1$, была построена схема $S_{n}$, реализующая систему $K_{n}$ со сложностью

$$
L\left(S_{n}\right)=3(2 n-1) 2^{n} .
$$

Н. А. Шкаликовой [4] показано, что $L\left(K_{n}\right) \asymp n 2^{n}$ при $n \rightarrow \infty$. Таким образом Н. А. Шкаликовой было установлено, что построенные С. С. Кравцовым схемы $S_{n}$ оптимальны по порядку роста сложности.

Легко подсчитать, что активность построенных в [1] оптимальных по порядку роста сложности схем $S_{n}$ дается соотношениями

$$
E_{\max }\left(S_{n}\right)=E_{\mathrm{m}}\left(S_{n}\right) \asymp n 2^{n}
$$

Однако оказывается, что эти схемы не оптимальны по порядку роста активности и в действительности порядки роста функций $E_{\max }\left(K_{n}\right)$ и $E_{\mathrm{m}}\left(K_{n}\right)$ существенно ниже. Имеет место следующее утверждение.

Теорема 1. При $n \rightarrow \infty$

$$
E_{\max }\left(K_{n}\right) \asymp E_{\mathrm{cp}}\left(K_{n}\right) \asymp 2^{n} .
$$

Доказательство теоремы разобьем на две леммы. 


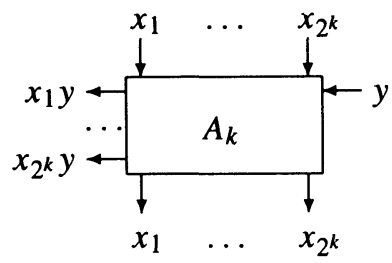

Рис. 6.

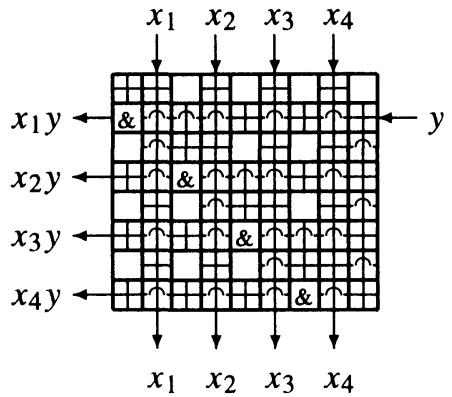

Рис. 7.

Лемма 1. Существует последовательность схем $W_{n}$, реализующих системы $K_{n}$ такая, что при $n \rightarrow \infty$

$$
E_{\text {max }}\left(W_{n}\right) \lesssim 5 \cdot 2^{n}
$$

Доказательство. Для построения схемы $W_{n}$ используем два экземпляра схем $S_{k}$, построенных в [1], и вспомогательные схемы $A_{k}$.

Схема $A_{k}$ реализует умножение $2^{k}$ входов $x_{1}, \ldots, x_{2^{k}}$ на выделенный вход $y$. Расположение входов и выходов этой схемы показано на рис. 6 . Строение схем $A_{k}$ можно понять на примере схемы $A_{2}$, которая изображена на рис. 7. Легко подсчитать, что

$$
m\left(A_{k}\right)=2 \cdot 2^{k}, \quad d\left(A_{k}\right)=2 \cdot 2^{k}+1 .
$$

Схема $S_{k}$ реализует систему всех элементарных конъюнкций $k$ переменных. Расположение входов и выходов этой схемы показано на рис. 8. Для схем $S_{k}$ в [1] показано, что

$$
m\left(S_{k}\right)=2 k-1, \quad d\left(S_{k}\right)=3 \cdot 2^{k}
$$

Теперь построим схему $W_{n}$. Положим $p=\lfloor n / 2\rfloor, q=\lceil n / 2\rceil$. При построении будем использовать две подсхемы $S_{p}$ и $S_{q}$. Обе эти подсхемы повернем на угол $\pi / 2$, а 


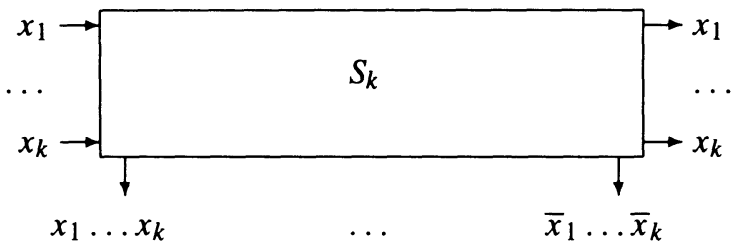

Рис. 8.

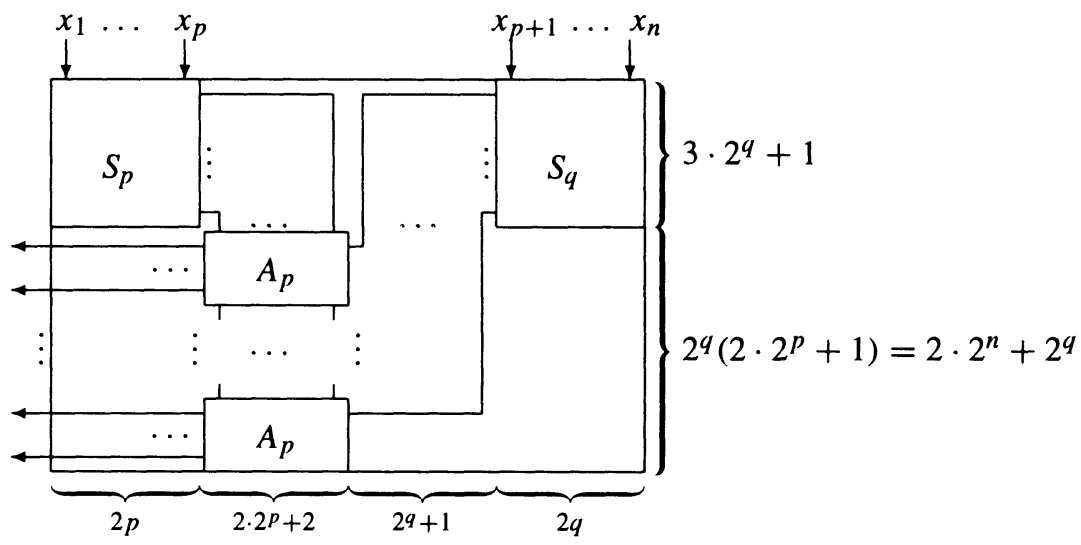

Рис. 9.

первую из них еще отобразим зеркально относительно вертикальной оси (из определения схемы видно, что это не отразится на ее функционировании). Также для построения схемы $W_{n}$ будем использовать $2^{q}$ подсхем $A_{p}$. Чтобы исключить паразитное взаямодействие различных подсхем, между ними требуется разместить изоляцию, обеспечивающую лишь необходимое взаимодействие различных подсхем. Изоляцией будем называть цепочки коммутационных элементов, в которых проводящие элементы стоят только в местах, соответствующих выходам подсхемы, а во всех остальных местах стоят пустые (изоляционные) элементы. Строение схемы $W_{n}$ показано на рис. 9. Изоляция на этом рисунке не показана явно, но учтена во всех указанных на рисунке размерах.

Подсчитаем величину $E_{\max }\left(W_{n}\right)$. Как было указано выше, $E_{\max }\left(S_{k}\right) \asymp k 2^{k}$, следовательно, вклад $S_{p}$ и $S_{q}$ в активность всей схемы будет пренебрежимо мал. Заметим, что при любом входном наборе лишь на одном выходе каждой из схем $S_{p}$ и $S_{q}$ будет реализоваться значение 1 . Соответственно, активность на проводах будет асимптотически не больше, чем $2 \cdot 2 \cdot 2^{n}=4 \cdot 2^{n}$. У той из подсхем $A_{p}$, на которую приходит единица от подсхемы $S_{q}$, будет активность $E_{\max }\left(A_{p}\right) \sim 2^{n}$, у остальных подсхем $A_{p}$ активность будет пренебрежимо мала (вклад в активность вертикального провода с единицей от схемы $S_{p}$, проходящего через все схемы $A_{p}$, мы уже посчитали выше). Таким образом, получим, что $E_{\max }\left(W_{n}\right) \lesssim 5 \cdot 2^{n}$

Замечание 1. Легко подсчитать, что $L\left(W_{n}\right) \asymp 2^{3 n / 2}$. 


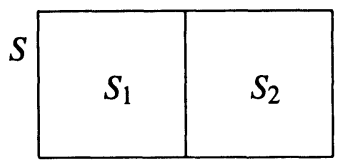

Рис. 10.

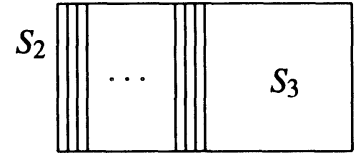

Рис. 11.

Лемма 2. Для любой последовательности схем $S^{n}$, реализуючих системы $K_{n}$, при $n \rightarrow \infty$ выполняется соотночение

$$
E_{\mathrm{m}}\left(S^{n}\right) \geqslant \frac{1}{50} 2^{n}(1+o(1))
$$

Доказательство. Пусть $S$ - произвольная схема, реализующая систему $K_{n}$ при некотором $n, n \geqslant 1$. Расположим схему $S$ таким образом, чтобы на ее горизонтальных сторонах было не меньше половины всех ее выходов (то есть не меньше чем $2^{n-1}$ выходов). Тогда мы можем разбить схему $S$ на две подсхемы $S_{1}$ и $S_{2}$ (рис. 10) так, чтобы в каждой из подсхем было не меньше чем $2^{n-1}-2$ выходов схемы $S$. Без ограничения общности будем считать, что в подсхеме $S_{2}$ не больше половины входов схемы $S$ (иначе повернем схему $S$ на $\pi$ ).

В дальнейшем будем называть сечением схемы всякую вертикальную цепочку ячеек.

Рассмотрим два случая:

(1) горизонтальная сторона подсхемы $S_{2}$ не меньше чем $\left[2^{n} / 20\right\rfloor+1$;

(2) горизонтальная сторона подсхемы $S_{2}$ меньше чем $\left\lfloor 2^{n} / 20\right\rfloor+1$.

В первом случае рассмотрим подсхему $S_{2}$. Построим в ней $\left\lfloor 2^{n} / 20\right\rfloor$ сечений как показано на рис. 11.

В оставшейся подсхеме $S_{3}$ будет не менее чем

$$
2^{n-1}-2-2\left\lfloor 2^{n} / 20\right\rfloor \geqslant \frac{2}{5} 2^{n}-4
$$

выходов схемы $S$ и не более чем $n / 2$ входов схемы $S$.

Для каждого построенного сечения $\alpha$ рассмотрим схему $S_{3}^{\alpha}$, образованную расширением схемы $S_{3}$ до сечения $\alpha$ так, чтобы ячейки сечения были входами схемы $S_{3}^{\alpha}$ (то есть объявим их входами схемы $S_{3}^{\alpha}$ наряду с имеющимися в ней входами исходной схемы $S)$. Здесь мы подразумеваем, что значения, реализуемые на выходах элементов сечения $\alpha$, подаются на входы схемы $S_{3}^{\alpha}$. Как и в схеме $S_{3}$, в схеме $S_{3}^{\alpha}$ будет не меньше чем $(2 / 5) 2^{n}-4$ выходов схемы $S$, и следовательно, будет не меньше чем (2/5) $2^{n}-4$ различных выходных наборов (так как каждый выход соответствует определенной конъюнкции, на каждом выходе должна реализовываться 1 (при определенном входном наборе), при этом на всех остальных выходах должны реализовываться 0). Очевидно, что различные 


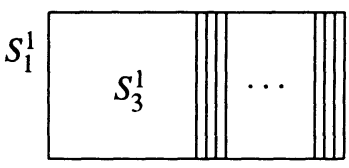

Рис. 12.

выходные наборы могут получаться только при различных входных наборах. Значит, у схемы $S_{3}^{\alpha}$ будет не меньше чем $(2 / 5) 2^{n}-4$ различных входных наборов.

Входной набор схемы $S_{3}^{\alpha}$ состоит из не более чем $n / 2$ входов схемы $S$ и ячеек сечения $\alpha$. Значит, не более чем $2^{n / 2}$ входных наборов могут содержать в сечении $\alpha$ одни только нули. Соответственно, не менее чем

$$
\frac{2}{5} 2^{n}-4-2^{n / 2}=\frac{2}{5} 2^{n}(1+o(1))
$$

входных наборов будут содержать в сечении $\alpha$ хотя бы одну единицу (полагаем, что $n \rightarrow \infty)$.

Суммируя по всем построенным сечениям и по всем входным наборам схемы $S$ и затем усредняя по всем этим наборам, получим, что

$$
E_{\mathrm{m}}(S) \geqslant \frac{1}{2^{n}} \frac{2^{n}}{20} \frac{2}{5} 2^{n}(1+o(1))=\frac{1}{50} 2^{n}(1+o(1)) .
$$

Во втором случае (если горизонтальная сторона подсхемы $S_{2}$ меньше чем $\left\lfloor 2^{n} / 20\right\rfloor+1$ ) разобьем схему $S$ на подсхемы $S_{1}^{1}$ и $S_{2}^{1}$ так, чтобы у подсхемы $S_{2}^{1}$ горизонтальная сторона была равна $\left\lfloor 2^{n} / 20\right\rfloor+1$. Заметим, что в подсхеме $S_{2}^{1}$ будет не менее чем $2^{n-1}-2$ выходов схемы $S$ (так как схема $S_{2}^{1}$ образована путем расширения схемы $S_{2}$ ). Поэтому, если в подсхеме $S_{2}^{1}$ не более чем $n / 2$ входов схемы $S$, то этот случай полностью аналогичен первому случаю.

Рассмотрим случай, когда в подсхеме $S_{2}^{1}$ больше чем $n / 2$ входов схемы $S$ (и, следовательно, в подсхеме $S_{1}^{1}$ меньше чем $n / 2$ входов схемы $S$ ). Изначально на горизонтальных сторонах схемы $S$ было не менее чем $2^{n-1}$ выходов схемы $S$. Так как горизонтальная сторона подсхемы $S_{2}^{1}$ равна $\left\lfloor 2^{n} / 20\right\rfloor+1$, на горизонтальных сторонах подсхемы $S_{1}^{1}$ не менее чем

$$
2^{n-1}-2-2\left\lfloor 2^{n} / 20\right\rfloor \geqslant \frac{2}{5} 2^{n}-4
$$

выходов схемы $S$. Аналогично первому случаю построим $\left\lfloor 2^{n} / 10\right\rfloor$ сечений (рис. 12).

Заметим, что в оставшейся подсхеме $S_{3}^{1}$ будет не менее чем

$$
\frac{2}{5} 2^{n}-4-2\left\lfloor\frac{2^{n}}{10}\right\rfloor \geqslant \frac{2^{n}}{5}-6
$$

выходов схемы $S$ (по крайней мере на горизонтальных сторонах найдется такое количество). Аналогично первому случаю получим, что при $n \rightarrow \infty$ выполняется соотношение

$$
E_{\mathrm{m}}(S) \geqslant \frac{1}{2^{n}} \frac{2^{n}}{10} \frac{2^{n}}{5}(1+o(1))=\frac{1}{50} 2^{n}(1+o(1))
$$

Теорема доказана. 


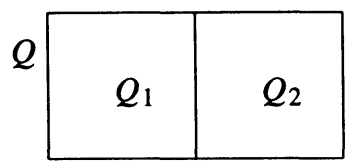

Рис. 13.

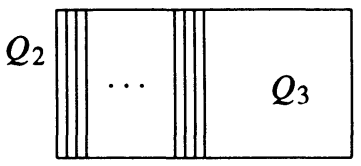

Рис. 14.

Теорема 1 дает ответ на вопрос о максимальной и средней активности системы $K_{n}$. Построенные выше схемы $W_{n}$ реализуют эти системы с оптимальной по порядку роста активностью. Как было сказано ранее, сложност. этих схем дается соотношением $L\left(W_{n}\right) \asymp 2^{3 n / 2}$. В соответствии с результатом Н. А. Шкаликовой [4], $L\left(K_{n}\right) \asymp n 2^{n}$, то есть схемы $W_{n}$ не оптимальны по порядку роста сложности. В связи с этим возникает вопрос о возможности одновременной минимизации порядков роста сложности и активности. Ответ на аналогичный вопрос для обычных схем из функциональных элементов, реализующих (одну) произвольную функщию $n$ переменных, был дан О. М. Касим-Заде [7]. Он показал, что при указанных условиях возможно одновременное достижение оптимального порядка роста мощности и асимптотики сложности.

Однако в нашем случае это оказывается не так.

Теорема 2. При $n \rightarrow \infty$ для любой последовательности схем $Q^{n}$, реализующих системы $K_{n}$ с минимальной по порядку роста сложностью (то есть удовлетворяющих соотношению $\left.L\left(Q^{n}\right) \asymp n 2^{n}\right)$ выполняются соотношения

$$
E_{\mathrm{m}}\left(Q^{n}\right) \geq n 2^{n}, \quad E_{\max }\left(Q^{n}\right) \geq n 2^{n} .
$$

Доказательство. Пусть $Q^{n}$ - последовательность схем, реализующих системы $K_{n}$, $n \rightarrow \infty$. Как показано в [4], любая схема, реализующая систему $K_{n}$ с оптимальной по порядку роста сложностью, имеет одну сторону (пусть это будет горизонтальная сторона) размера $d\left(Q^{n}\right) \asymp 2^{n}$, а другую сторону (соответственно, это будет вертикальная сторона) размера $m\left(Q^{n}\right) \asymp n$. Отсюда следует, что существуют константы $c_{1}$ и $c_{2}$ такие, что ширина (размер вертикальной стороны) схем $Q^{n}$ удовлетворяет неравенствам $c_{1} n \leqslant m\left(Q^{n}\right) \leqslant c_{2} n$.

Пусть $Q-$ одна из схем, реализующая систему $K_{n}$ при некотором $n, n \geqslant 1$. Разобьем схему $Q$ на две подсхемы $Q_{1}$ и $Q_{2}$ (рис. 13) так, чтобы в каждой из подсхем было не менее чем $2^{n-1}-2$ выходов схемы $Q$.

Без ограничения общности будем считать, что в подсхеме $Q_{2}$ не более чем $n / 2$ входов схемы $Q$. Аналогично доказательству леммы 2 построим $2^{n-3}$ сечений в подсхеме $Q_{2}$ (рис. 14).

В оставшейся подсхеме $Q_{3}$ будет не менее чем $2^{n-1}-2-2 \cdot 2^{n-3}=2^{n-2}-2$ выходов схемы $Q$. Аналогично доказательству леммы 2 для каждого сечения $\alpha$ рассмотрим схему $Q_{3}^{\alpha}$. У нее должно быть не менее чем $2^{n-2}-2$ различных входных наборов, каждый из которых состоит из не более чем $n / 2$ значений входов схемы $Q$ и значений, реализуемых 
в ячейках сечения $\alpha$. Таким образом, различных входных наборов ровно с $k$ единицами в сечении будет не более, чем $\left(\begin{array}{c}m(S) \\ k\end{array}\right) 2^{n / 2}$, где $\left(\begin{array}{l}n \\ k\end{array}\right)$ - биномиальный коэффициент. Воспользуемся известной оценкой $[9,10]$ для биномиальных коэффициентов

$$
\sum_{i=0}^{s}\left(\begin{array}{l}
n \\
i
\end{array}\right) \leqslant 2^{H(s / n) n},
$$

справедливой при условии, что $s / n \leqslant 1 / 2$, где $H(x)-$ функщия двоичной энтропии, то есть $H(x)=-x \log _{2} x-(1-x) \log _{2}(1-x)$ при $0<x<1$ и $H(0)=H(1)=0$.

Зададимся произвольным числом $\beta, 0 \leqslant \beta \leqslant 1 / 2$. Тогда в соответствии с указанной оценкой число входных наборов с не более чем $\beta c_{1} n$ единицами в сечении $\alpha$ не превосходит величины $2^{H\left(\beta c_{1} n / m(S)\right) m(S)} 2^{n / 2} \leqslant 2^{H(\beta) c_{2} n} 2^{n / 2}$.

Для всякой константы $c_{2}$ число $\beta$ может быть выбрано настолько малым, чтобы выполнялось неравенство $H(\beta) c_{2}<1 / 2$, и следовательно, $2^{H(\beta) 2_{2} n} 2^{n / 2}=o\left(2^{n}\right)$. Тогда различных входных наборов схемы $Q_{3}^{\alpha}$ с более чем $\beta c_{2} n$ единицами, будет не менее чем $2^{n-2}(1+o(1))$.

Суммируя по всем построенным сечениям и по всем входным наборам схемы $Q$ и затем усредняя по всем этим наборам, получим, что

$$
E_{\mathrm{m}}(Q) \geqslant \frac{1}{2^{n}} 2^{n-3} \beta c_{2} n 2^{n-2}(1+o(1)) \text {. }
$$

При фиксированном $\beta$ и $n \rightarrow \infty$ отсюда следует, что $E_{\mathrm{m}}\left(Q^{n}\right) \geq n 2^{n}$. Осталось учесть, что $E_{\max }\left(Q^{n}\right) \geqslant E_{\mathrm{m}}\left(Q^{n}\right)$. Теорема доказана.

В заключение, хочу выразить глубокую благодарность О. М. Касим-Заде за постановку задачи и постоянное внимание к работе.

\section{Список литературы}

1. Кравцов С. С., О реализации функций алгебры логики в одном классе схем из функциональных и коммутационных элементов. Проблемы кибернетики (1967) 19, 285-292.

2. Альбрехт А., О схемах из клеточных элементов. Проблемы кибернетики (1978) 33, 209-214.

3. Шкаликова Н. А., О сложности реализации некоторых функций клеточными схемами. Сборник работ по математической кибернетике (1976) 1, 102-115.

4. Шкаликова Н. А., О реализации булевых функций схемами из клеточных элементов. Матем. вопросы кибернетики (1989) 2, 177-197.

5. Вайнцвайг М. Н., О мощности схем из функциональных элементов. Докл. АН CCCP (1961) 139, №2, 320-323.

6. Касим-Заде О. М., Об одной мере сложности схем из функциональных элементов. Проблемы кибернетики (1981) 38, 117-179.

7. Касим-Заде О. М., Об одновременной минимизации сложности и мощности схем из функциональных элементов. Проблемы кибернетики (1978) 33, 215-220.

8. Лупанов О. Б., О синтезе некоторых управляющих систем. Проблемы кибернетики (1963) 10, 63-97.

9. Мак-Вильямс Ф., Слоэн Н., Теория кодов, исправляющих ошибки. Радио и связь, Москва, 1979.

10. Яблонский С. В., Лупанов О. Б. (ред.) Дискретная математика и математические вопросы кибернетики, т. 1. Наука, Москва, 1974. 